\title{
Continuity, Coordination, and Transitions of Care for Patients with Serious and Advanced Illness: A Systematic Review of Interventions
}

\author{
Sydney M. Dy, MD, MSc, ${ }^{1,2}$ Colleen Apostol, MSN, RN, Kathryn A. Martinez, PhD, MPH, ${ }^{4}$ \\ and Rebecca A. Aslakson, MD, MSc ${ }^{2}$
}

\begin{abstract}
Objectives: Continuity, coordination, and transitions of care are key to high-quality medical care for patients with serious and advanced illness. We conducted a systematic review to evaluate the impact of interventions targeting these areas in this population.

Methods: We searched PubMed, CINAHL, PsycINFO, Cochrane, and DARE from 2000 through 2011. We included prospective controlled studies targeting continuity, coordination, and transitions for patients with advanced illness that reported patient centered outcomes. Of 13,014 citations, 23 studies met inclusion criteria. Two investigators extracted and checked data on population, interventions, methods, outcomes, and methodological quality.

Results: Four of the six studies evaluating patient satisfaction $(67 \%)$ and four of the six studies evaluating caregiver satisfaction $(67 \%)$ showed statistically significant improvements in these outcomes in the intervention compared to the control group. Only three of the nine studies (33\%) measuring quality of life and five of the 16 $(31 \%)$ measuring health care utilization showed improvement. Results were similar across different types of interventions.

Conclusions: Many studies were limited by methodologic issues such as use of measurement tools not developed for patients with advanced disease and small sample size. Interventions and outcomes were too heterogeneous for meta-analysis. We found moderate evidence that interventions targeting continuity, coordination, and transitions in patients with advanced and serious illness improve patient and caregiver satisfaction, but low evidence for other outcomes. Further research is needed on how to target these domains for outcomes such as health care utilization.
\end{abstract}

\section{Introduction}

C ONTINUITY, COORDINATION, AND TRANSITIONS of care are key domains for improving quality of care for patients with advanced and serious illness. These three concepts are key standards for quality palliative care ${ }^{1}$ and key determinants of satisfaction with care for patients at the end of life. ${ }^{2,3}$ Continuity can be defined as the exchange of knowledge and the relationships between providers and patients or families, or between providers and/or provider groups; an example of improving continuity is standard assessment of palliative care needs. Coordination can be defined as the alignment of care across providers and settings; an example is a palliative care nurse coordinating care among the radiation, medical, and surgical oncologists for a patient. Finally, transitions of care generally refers to care across settings or providers of care. Improving transitions in palliative care often refers to facilitation, when appropriate, of patient care goals to more comfort-oriented care; an example is helping appropriate patients transition to hospice care.

Due to the complexity of care for patients with advanced and serious illness, these domains of care are often not well addressed. For example, despite the benefits of hospice care on improved end-of-life outcomes such as family satisfaction with care, ${ }^{4}$ timely transition to hospice care frequently does not occur in the United States. In 2009, less than half of

\footnotetext{
${ }^{1}$ Bloomberg School of Public Health, ${ }^{2}$ School of Medicine, Johns Hopkins University, Baltimore, Maryland.

${ }^{3}$ The Johns Hopkins Medical Institutions, Johns Hopkins Hospital, Baltimore, Maryland. ${ }^{4}$ Division of General Medicine, University of Michigan, Ann Arbor, Michigan.

Accepted February 8, 2013.
} 
patients who died ever received hospice care. ${ }^{1}$ Specific types of quality improvement interventions have been developed and evaluated to meet these needs, such as the inclusion of an additional provider (a nurse or social worker) to provide case management to specifically target these domains, or the use of specialized palliative care service teams. ${ }^{5}$

Although previous systematic reviews have addressed specific types of interventions targeting continuity and coordination, such as specialized palliative care, ${ }^{6,7}$ these reviews have not addressed the full spectrum of types of interventions targeting these domains or evaluated the effectiveness of different components of interventions. We therefore conducted a systematic review to investigate if interventions focusing on continuity, coordination, and transitions of care for patients with advanced and serious illness improve different patient and caregiver centered outcomes, including quality of life, satisfaction, and health care utilization, and to identify which components are associated with effective interventions.

\section{Methods}

As part of a larger systematic review ${ }^{8}$ on interventions to improve health care in patients with advanced and serious illness, we included studies that had a primary focus on continuity, coordination, and transitions of care.

\section{Definition of studies targeting improvements in continuity, coordination, and transitions of care}

For the definition of continuity, coordination, and transitions, we included interventions that targeted improving the relationship between patients or families and providers, the relationship between providers (e.g., developing interdisciplinary teams), and on improving information exchange (e.g., improving patient assessment in palliative care domains) (continuity); improving alignment of care across providers and setting (e.g., case management) (coordination); and improving care across settings or providers of care (e.g., facilitating hospice referrals) (transitions).

\section{Intervention components}

Because of the heterogeneity of the interventions, we classified interventions by five key components (studies could have multiple components). Patient/family/caregiver involvement refers to an intervention focused directly on them, for example through education. Coordination refers to interventions which utilized an additional provider to coordinate health care. Care plans refers to interventions that incorporated care plans or order sets with directions to follow for providers (e.g., comfort care orders, flow sheets). Palliative care specialist refers to interventions employing any type of specialist palliative care providers. Finally, additional assessments refers to any assessment (e.g., questionnaires, predefined assessment lists) that was added to usual care as part of the intervention.

\section{Data sources}

We searched the electronic databases PubMed, CINAHL, PsycINFO, Cochrane, and DARE (details in Appendix A) from 2000 through December 2011. We reviewed reference lists of included articles for potentially relevant studies. $\mathrm{MeSH}$ terms included palliative care and quality improvement; keywords included cancer, terminally ill, hospice care, patient care planning, and quality assurance (detailed search strategy is in Appendix A). We also identified systematic reviews that might contain relevant articles and reviewed these reference lists as well.

\section{Study eligibility criteria, participants, and interventions}

We included randomized controlled trials and prospective controlled studies (prospective before-after studies or studies with a nonrandomized control group). We included all ages of patients and all settings, including inpatient facilities, outpatient care, nursing homes and hospices. Specific inclusion criteria were that studies must be composed primarily of seriously ill patients or those with advanced disease who were unlikely to be cured, recover, or stabilize (definition adapted from the National Consensus Project). ${ }^{9}$ Since many studies combined populations (e.g., cancer survivors, patients with early-stage disease, and patients with advanced disease), we included studies where more than $50 \%$ of the included population would fit the above definition. When results were reported separately, we abstracted only the results for the relevant population.

We focused on key patient-centered outcomes related to the domains of interest, including patient satisfaction and quality of life (QOL), family or caregiver burden and/or satisfaction and/or QOL, health care utilization (e.g., potentially avoidable utilization such as admissions, length of stay, diagnostic interventions), location of death (e.g., home versus hospital), hospice referral, and do-not-resuscitate and advanced directive status (see full report for details). ${ }^{2}$ We excluded studies that did not report patient-centered outcomes (e.g., studies of staff knowledge). We excluded studies published before 2000, since both interventions to improve health care and palliative care practice have changed substantially since that time, and the pre-2000 literature has been well-addressed in earlier reviews. ${ }^{10}$

\section{Study appraisal and synthesis methods}

Two members of the review team independently assessed each title and abstract using DistillerSR, an online systematic review software program. Disagreements were resolved by the reviewers or by consensus. The full text of eligible articles was retrieved and evaluated by two reviewers for inclusion criteria and to identify the target of the intervention.

One reviewer recorded details about study design, interventions and their components, study population, outcome measures, results (including study statistics), and study quality (using risk of bias assessment), ${ }^{11}$ and a second reviewer checked data for accuracy. For study quality, we assessed randomization generation, concealment of allocation, masking of patient, personnel and outcome assessors, how incomplete outcome data was addressed and selective outcome reporting. All evidence was graded for the strength of the best available evidence, including grading study quality with the risk of bias in relevant studies, using the Grade of Recommendations Assessment, Development, and Evaluation (GRADE) Working Group criteria adapted by the Agency for Healthcare Research and Quality. ${ }^{12}$ For classification of overall quality, we started with high quality for RCTs and downgraded a study if there was a lack of documentation of important factors (e.g., blinding, concealment); for non-RCTs we started with low quality and upgraded a study if appropriate. 
We used descriptive statistics to summarize our findings across the entire set of studies, and to describe results by outcome and by components of the intervention. We could not perform meta-analysis due to intervention and outcome heterogeneity. ${ }^{13}$

\section{Results}

The literature search identified 13,014 unique citations. During the title and abstract review process, we excluded 12,567 abstracts that did not meet one or more of the eligibility criteria (see Figure 1), and at article review, we excluded an additional 351 articles that did not meet one or more of the eligibility criteria. Ninety-three articles were eligible for the systematic review. There were 23 studies described in 26 articles that we categorized as continuity, coordination, or transitions of care. All but two of the included studies compared an intervention to usual care; these two studies compared the same intervention with and without information exchange between providers.

Twelve studies were RCTs, four had concurrent controls, and seven were prospective before-after studies. Five studies were conducted in an inpatient setting, four in a home care setting, four in an ambulatory setting, three in nursing homes, one in hospice, one in a rehabilitation unit, and five in mixed settings. Fifteen studies (65\%) were multicenter studies. One study reported only within group comparisons and, therefore, the outcomes are not further analyzed here. ${ }^{14}$ All studies were in adults, with a mean age (by study) between 62 and 84 . Most included patients with mixed illnesses (nine studies) or cancer patients only (seven studies) (see Table 1). In 11 studies, the intervention was provided by the existing staff. In nine studies, the intervention was provided by an external team or consultant, and in three studies both approaches were used.

The results section is organized as follows: summary of the evidence for each of the patient/caregiver-centered outcomes; summary for different intervention components for patient/ caregiver-centered outcomes overall; summary for health care utilization; and summary for other outcomes.

\section{Evidence for patient/caregiver-centered outcomes: Patient or caregiver QOL and satisfaction and caregiver burden}

Nine studies evaluated QOL of patients. ${ }^{15-23}$ Five of these nine studies were of good quality and all but one were RCTs. Only three studies (33\%) showed a statistically significant improvement on QOL of patients with the intervention compared to the control group (see Table 1). ${ }^{16,17,23}$

Seven studies assessed patient satisfaction; ${ }^{17,21,22,24-27}$ four were RCTs. The overall quality ranged from poor to good. One trial reported QOL for within group comparison only and was therefore not included in the analysis. ${ }^{24}$ Of the other six studies, four $(67 \%)$ showed statistically significant benefits from the intervention. ${ }^{21,24,26,27}$ Three studies measured both patient satisfaction and QOL; ${ }^{17,21,22}$ two studies found

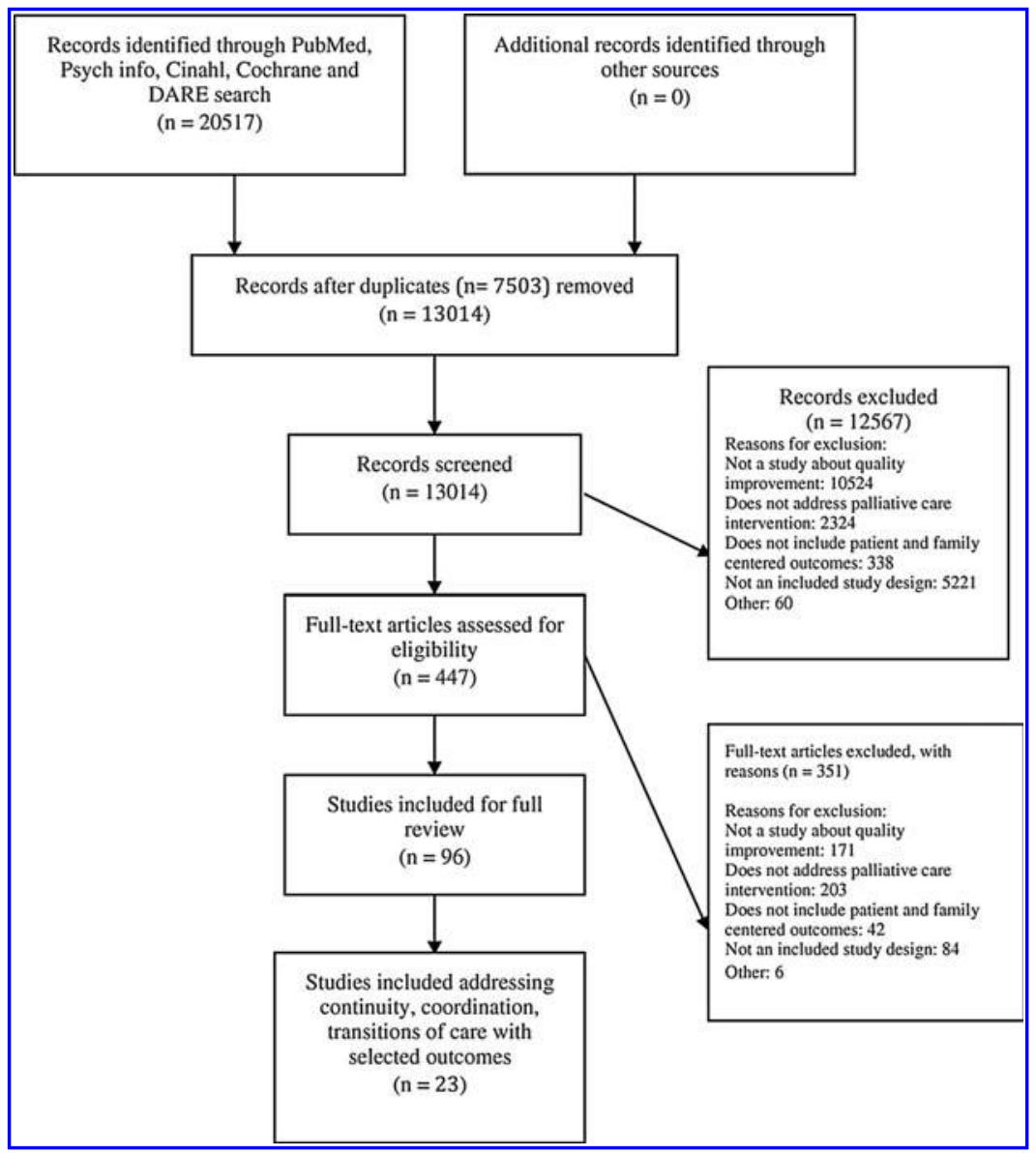

FIG. 1. Article flow for systematic review. 
significant improvements for only one of the outcomes, and the remaining study found no effect on either outcome. ${ }^{22}$

Six studies reported caregiver satisfaction; ${ }^{17,27-30,34}$ three were RCTs. Four of these studies (67\%), including the three RCTs, showed statistically significant improvements in the intervention groups (see Table 2). ${ }^{17,27,29,34}$ Three studies reported caregiver burden ${ }^{17,20,28}$ (two RCTs); none showed a significant difference between the groups. Only one lowquality study reported caregiver QOL, ${ }^{27}$ and showed statistically significant improvement. Among the three studies evaluating at least two of these three caregiver outcomes, two had conflicting findings for the different outcomes and one found that neither caregiver burden nor satisfaction showed a statistically significant improvement with the intervention. $^{28}$

Strength of evidence was moderate for patient and caregiver satisfaction and low for other outcomes.

\section{Evidence for different intervention components for patient/caregiver-centered outcomes}

Table 2 illustrates the components of each intervention and if the intervention was statistically significant and improved outcomes compared to the control group. Seven of the nine (78\%) studies including the component of patient and/or family involvement that measured these outcomes showed a statistically significant improvement with the intervention compared to the control group on at least one of the outcomes of patient or family QOL, satisfaction or burden (results for medium- and high-quality studies only: six of the eight studies $(75 \%)$ showed a significant effect). Nine of the twelve studies $(75 \%)$ using additional patient assessments found a significant improvement on at least one of these outcomes (only medium- and high-quality studies: eight of 10 studies $(80 \%))$. Six of the nine $(67 \%)$ studies using coordination showed improvement on at least one QOL or satisfaction outcome (all were medium- or high-quality studies). Three of the five $(60 \%)$ studies using palliative care specialists found a significant improvement on at least one of the outcomes (all were medium- and high-quality studies). Five of the nine $(56 \%)$ studies using care plans or order sets showed an improvement on at least one of these outcomes (results for medium- and high-quality studies only: four of six studies $(67 \%))$.

\section{Evidence for different intervention elements for studies that measured health care utilization, e.g., admissions, length of stay}

In total, 16 studies reported statistics on health care utilization $^{15-17,21-28,32-37}$ (one reported health care utilization but no statistical testing), primarily length of stay or different types of admissions (a few studies assessed utilization of diagnostic or therapeutic interventions). Eight of these studies were RCTs. Five of these studies (31\%) found a statistically significant effect in favor of the intervention compared to the control group.

Four of the eleven (36\%) studies using care plans or order sets that measured this outcome showed decreased utilization (only medium- and high-quality studies: two of seven studies; $29 \%$ ). Five of the fifteen (33\%) using additional assessments showed decreased utilization (only medium- and high-quality studies: three of 10 studies; 30\%). Three of the ten $(30 \%)$ studies with patient and/or family involvement that measured this outcome showed a significant decrease in health care utilization for the intervention (only mediumand high-quality studies: two of eight; $25 \%$ ). In two of the ten $(20 \%)$ studies using coordination, patients had significantly lower health care utilization with the intervention (only medium- and high-quality studies: two of nine studies; $22 \%$ ). None of the five studies using palliative care specialists showed a significant effect. Strength of evidence was low for utilization.

\section{Other outcomes}

Location of death was reported as an outcome in six studies, $^{23,28,31,34-36}$ three of which were RCTs. In three of these studies $(50 \%),{ }^{23,31,36}$ patients in the intervention groups were more likely to die at home. Four studies reported enrollment in hospice, use of hospice services or placing patients on a comfort care plan (two were RCTs). ${ }^{23,32,34,37}$ Two (50\%) reported significant results in favor of the intervention. ${ }^{32}$

Four studies reported on do-not-resuscitate and advanced directive status ${ }^{15,26,35,38}$ as an outcome of the intervention. Two were RCTs. ${ }^{15,26}$ Two of the four (50\%) showed statistically significantly more do-not-resuscitate orders or advanced directives in the intervention group. ${ }^{15,35}$ Eight studies reported on overall symptom control; none of these studies showed an effect of the intervention. Strength of evidence was low for these outcomes.

\section{Discussion}

In this systematic review of interventions targeting continuity, coordination and transitions of care for patients with advanced and serious illness, in a mix of populations and settings, we found moderate strength of evidence only for the outcomes of patient and caregiver satisfaction. Strength of evidence was low for other outcomes evaluated in these studies, including patient quality of life, caregiver burden, health care utilization, location of death, and do not resuscitate orders or advance directives, and overall symptoms, although many of these outcomes were evaluated in only a few studies. The interventions that did improve outcomes were heterogeneous; most used components of coordination, patient and/or family involvement, and/or additional patient assessment. Results were similar across interventions using these different components of care.

Satisfaction is an important outcome as it reflects one of the primary goals of continuity-improved relationships and experience of care-measured from the perspective of the patient and family; our findings for patient and family satisfaction were similar. Some of the findings of this review are similar to those of previous reviews addressing subsets of this literature, particularly the effectiveness of specialized palliative care. $6,7,10,40-44$ The most recent review of specialized palliative care ${ }^{6}$ focused on RCTs through 2007 and the outcomes of quality of life, satisfaction, or costs. This review found that evidence was most consistent for the outcome of family satisfaction; as in our review, few studies evaluating quality of life or cost found evidence for these outcomes. The most recent review on specialized palliative care in cancer ${ }^{25}$ included a wider range of studies than in our review, including retrospective and uncontrolled studies, and found 


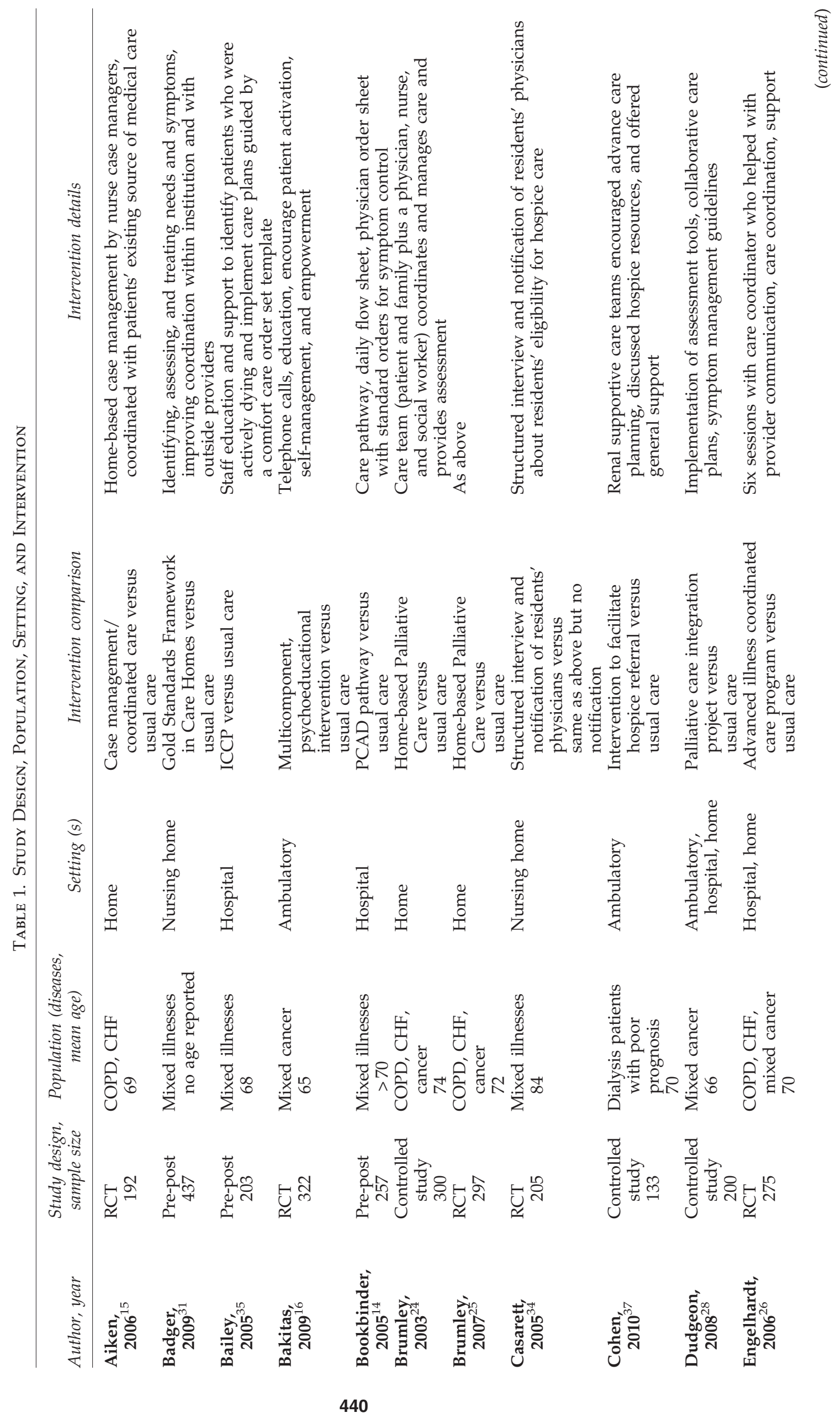




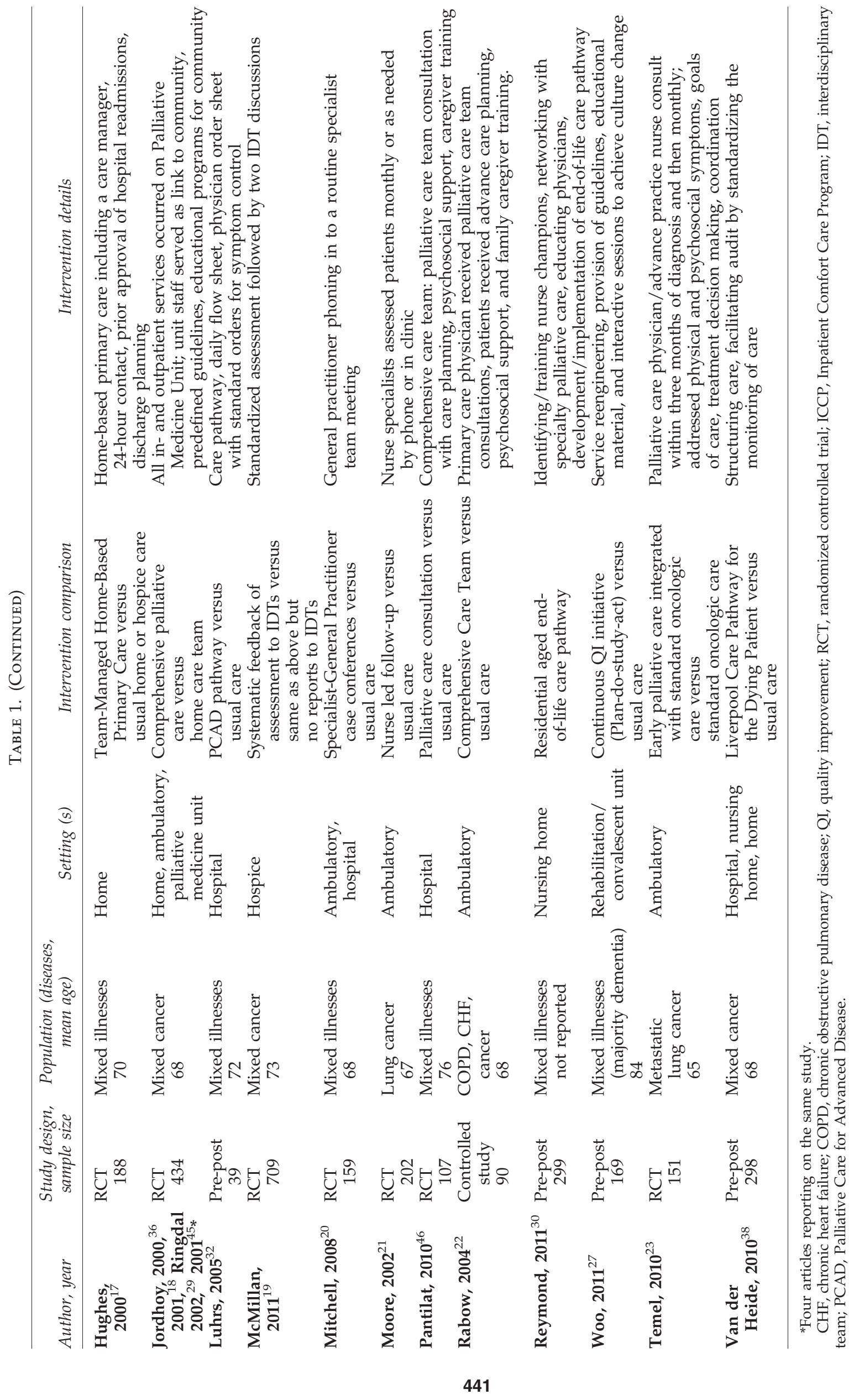




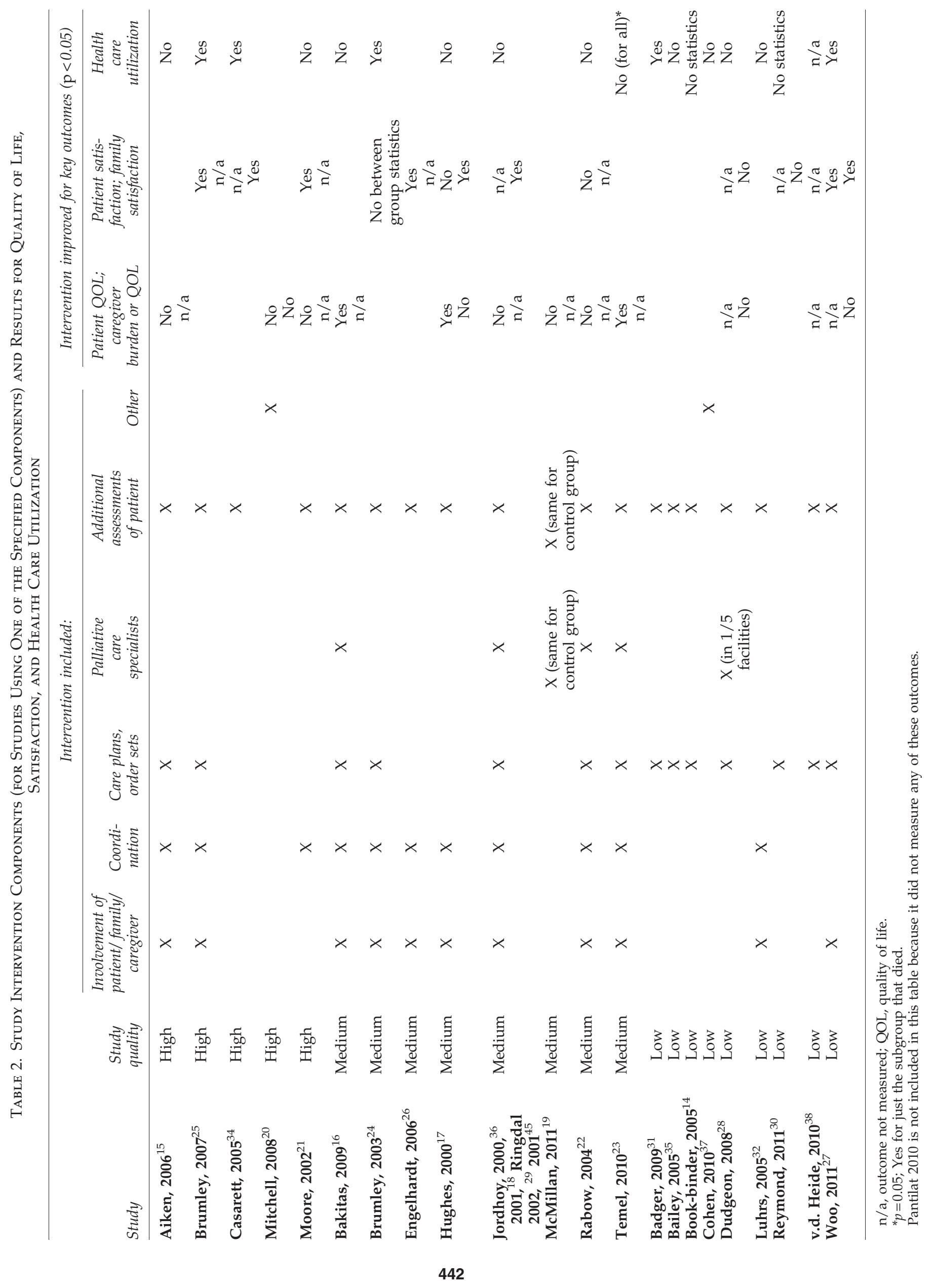


more evidence to support the outcomes of hospital admissions and satisfaction than quality of life.

Several older systematic reviews have addressed the target of continuity in related areas, although focusing on broader populations or only specific types of interventions. One previous review of case management in end-of-life care found mixed effects for outcomes (primarily health care utilization), ${ }^{10}$ and a review of improved coordination for supportive cancer care found little evidence to support portable medical records and mixed effects for palliative care-home care coordination. ${ }^{10}$

The studies targeting these domains had a number of limitations. Although we identified a few high-quality studies with consistent evidence across outcomes, in general, the quality of evidence was moderate to low. Many studies were limited by numerous methodological issues such as insufficient power for reported outcomes (particularly utilization), measuring outcomes not specifically targeted by the intervention, and using measurement tools (especially for quality of life outcomes) not specific for populations with advanced disease.

Our review also has several limitations. This review focuses on studies that we classified with a primary target of continuity, coordination, or transitions of care; studies focusing on other targets, such as communication or pain management, or on multiple targets, were addressed separately in the larger project. ${ }^{8}$ Meta-analyses were not possible as the interventions and outcomes were too heterogeneous. The comparison of different components of the interventions was also limited by study heterogeneity and by the relatively small number of studies for each component and outcome. Finally, we focused on prospective studies with the intent of limiting to higherquality studies, and did not address the substantial number of retrospective studies in this population evaluating health care utilization outcomes.

In conclusion, we found moderate strength of evidence for interventions targeting continuity, coordination, and transitions of care in patients with advanced and serious illness only for the outcomes of patient and family satisfaction. Future research should address other outcomes for which we found low strength of evidence, including health care utilization. Large multicenter trials are ongoing in the field of palliative care, which should improve the quality and quantity of current evidence. Ideally, future studies should use some of the instruments newly developed and validated for sensitivity to interventions in palliative care populations (particularly for quality of life); the use of more general instruments may explain why some interventions did not show an effect. Ideally, future interventions should specifically describe the targets of the intervention and define primary and secondary outcomes that best fit those targets. Finally, development of measurement instruments is still needed in some key areas, such as caregiver burden.

\section{Acknowledgments}

This project was funded under Contract No. HHSA-2902007-10061-I-EPC3 from the Agency for Healthcare Research and Quality, U.S. Department of Health and Human Services. The Agency for Healthcare Research and Quality reviewed contract deliverables for adherence to contract requirements and quality. The authors of this report are responsible for its content. Statements in the report should not be construed as endorsement by the Agency for Healthcare Research and Quality or the U.S. Department of Health and Human Services.

\section{Author Disclosure Statement}

No conflicting financial interests exist.

\section{References}

1. National Hospice and Palliative Care Organization: NHPCO Facts and Figures: Hospice Care in America. 2010. www.nhpco.org. (Last accessed, July 15, 2012.)

2. Dy SM, Shugarman LR, Lorenz KA, Mularski RA, Lynn J: A systematic review of satisfaction with care at the end of life. J Am Geriatr Soc 2008;56(1):124-129.

3. Back AL, Young JP, McCown E et al.: Abandonment at the end of life from patient, caregiver, nurse, and physician perspectives: Loss of continuity and lack of closure. Arch Intern Med 2009;169(5):474-479.

4. Teno JM, Clarridge BR, Casey V et al.: Family perspectives on end-of-life care at the last place of care. IAMA 2004;291(1):88-93.

5. Higginson IJ, Evans CJ: What is the evidence that palliative care teams improve outcomes for cancer patients and their families? Cancer J 2012;16(5):423-435.

6. Zimmermann C, Riechelmann R, Krzyzanowska M, Rodin G, Tannock I: Effectiveness of specialized palliative care: A systematic review. JAMA 2008;299(14):1698-709.

7. Higginson IJ, Evans CJ. What is the evidence that palliative care teams improve outcomes for cancer patients and their families? Cancer J 2010;16(5):423-435.

8. Dy SM, Aslakson R, Wilson RF, Fawole OA, Lau BD, Martinez KA, Vollenweider D, Apostol C, Bass EB: Interventions to Improve Health Care and Palliative Care for Advanced and Serious Illness. Closing the Quality Gap: Revisiting the State of the Science. Evidence Report No. 208. Prepared by the Johns Hopkins University Evidence-Based Practice Center under Contract 290-2007-10061-I. Agency for Healthcare Research and Quality Publication 12-E014-EF. Rockville, MD: Agency for Healthcare Research and Quality. 2012. www.effective healthcare.ahrq.gov/reports/final.cfm. (Last accessed March 7, 2013.)

9. National Consensus Project for Quality Palliative Care: Clinical Practice Guidelines for Quality Palliative Care, 2nd ed. 2009. www.nationalconsensusproject.org. (Last accessed July 15, 2012.)

10. Lorenz K, Lynn J, Morton SC, et al.: End-of-life care and outcomes. Evid Rep Technol Assess (Summ) 2004;(110):1-6.

11. Higgins JPT. Cochrane Handbook for Systematic Reviews of Interventions, version 5.0.2. The Cochrane Collaboration. 2009. www.cochrane.org.

12. AHRQ: Methods Guide for Effectiveness and Comparative Effectiveness Reviews. 2011. AHRQ Publication 10(11)-EHC063-EF. Rockville, MD: Agency for Healthcare Research and Quality.

13. Balshem $H$, Helfand $M$, Schunemann HJ, et al.: GRADE guidelines: 3. Rating the quality of evidence. J Clin Epidemiol 2011;64(4):401-406.

14. Bookbinder M, Blank AE, Arney E , et al.: Improving end-oflife care: Development and pilot-test of a clinical pathway. I Pain Symptom Manage 2005;29(6):529-543.

15. Aiken LS, Butner J, Lockhart CA, Volk-Craft BE, Hamilton G, Williams FG: Outcome evaluation of a randomized trial 
of the PhoenixCare intervention: Program of case management and coordinated care for the seriously chronically ill. I Palliat Med 2006;9(1):111-126.

16. Bakitas M, Lyons KD, Hegel MT, et al.: Effects of a palliative care intervention on clinical outcomes in patients with advanced cancer: The Project ENABLE II randomized controlled trial. JAMA 2009;302(7):741-749.

17. Hughes SL, Weaver FM, Giobbie-Hurder A, et al.: Effectiveness of team-managed home-based primary care: A randomized multicenter trial. JAMA 2000;284(22):2877-2885.

18. Jordhoy MS, Fayers P, Loge JH, Ahlner-Elmqvist M, Kaasa S: Quality of life in palliative cancer care: Results from a cluster randomized trial. J Clin Oncol 2001;19(18):3884-3894.

19. McMillan SC, Small BJ, Haley WE: Improving hospice outcomes through systematic assessment: A clinical trial. Cancer Nurs 2011;34(2):89-97.

20. Mitchell GK, Del Mar CB, O'Rourke PK, Clavarino AM: Do case conferences between general practitioners and specialist palliative care services improve quality of life? A randomised controlled trial. Palliat Med 2008;22(8):904-912.

21. Moore S, Corner J, Haviland J, et al.: Nurse led follow up and conventional medical follow up in management of patients with lung cancer: Randomised trial. BMJ 2002; 325(7373):1145.

22. Rabow MW, Dibble SL, Pantilat SZ, McPhee SJ. The comprehensive care team: A controlled trial of outpatient palliative medicine consultation. Arch Intern Med 2004;164(1):83-91.

23. Temel JS, Greer JA, Muzikansky A, et al.: Early palliative care for patients with metastatic non-small-cell lung cancer. NEJM 2010;363(8):733-742.

24. Brumley RD, Enguidanos S, Cherin DA: Effectiveness of a home-based palliative care program for end-of-life. J Palliat Med 2003;6(5):715-724.

25. Brumley R, Enguidanos S, Jamison P, et al.: Increased satisfaction with care and lower costs: Results of a randomized trial of in-home palliative care. J Am Geriatr Soc 2007;55(7): 993-1000.

26. Engelhardt JB, McClive-Reed KP, Toseland RW, Smith TL, Larson DG, Tobin DR: Effects of a program for coordinated care of advanced illness on patients, surrogates, and healthcare costs: A randomized trial. Am J Manag Care 2006; 12(2):93-100.

27. Woo J, Cheng JO, Lee J, et al.: Evaluation of a continuous quality improvement initiative for end-of-life care for older noncancer patients. J Am Med Dir Assoc 2011;12(2):105-113.

28. Dudgeon DJ, Knott C, Eichholz M, et al.: Palliative Care Integration Project (PCIP) quality improvement strategy evaluation. J Pain Symptom Manage 2008; 35(6):573-582.

29. Ringdal GI, Jordhoy MS, Kaasa S: Family satisfaction with end-of-life care for cancer patients in a cluster randomized trial. J Pain Symptom Manage 2002; 24(1):53-63.

30. Reymond L, Israel FJ, Charles MA: A residential aged care end-of-life care pathway (RAC EoLCP) for Australian aged care facilities. Aust Health Rev 2011;35(3):350-356.

31. Badger F, Clifford C, Hewison A, Thomas K: An evaluation of the implementation of a programme to improve end-oflife care in nursing homes. Palliat Med 2009;23(6):502-511.

32. Luhrs CA, Meghani S, Homel P, et al.: Pilot of a pathway to improve the care of imminently dying oncology inpatients in a Veterans Affairs Medical Center. J Pain Symptom Manage 2005;29(6):544-551.

33. Kitchener HC, Fletcher I, Roberts C, Wheeler P, Almonte M, Maguire P: The psychosocial impact of human papillomavirus testing in primary cervical screening: A study within a randomized trial. Int J Gynecol Cancer 2008;18(4):743-748.

34. Casarett D, Karlawish J, Morales K, Crowley R, Mirsch T, Asch DA: Improving the use of hospice services in nursing homes: A randomized controlled trial. JAMA 2005;294(2): 211-217.

35. Bailey FA, Burgio KL, Woodby LL, et al.: Improving processes of hospital care during the last hours of life. Arch Intern Med 2005;165(15):1722-1727.

36. Jordhoy MS, Fayers P, Saltnes T, Ahlner-Elmqvist M, Jannert M, Kaasa S: A palliative-care intervention and death at home: A cluster randomised trial. Lancet 2000;356(9233):888-893.

37. Cohen LM, Ruthazer R, Germain MJ: Increasing hospice services for elderly patients maintained with hemodialysis. I Palliat Med 2010;13(7):847-854.

38. van der Heide A, Veerbeek L, Swart S, van der Rijt C, van der Maas PJ, van Zuylen L: End-of-life decision making for cancer patients in different clinical settings and the impact of the LCP. J Pain Symptom Manage 2010;39(1):33-43.

39. Ferri CV, Pruchno RA: Quality of life in end-stage renal disease patients: Differences in patient and spouse perceptions. Aging Ment Health 2009;13(5):706-714.

40. Lorenz KA, Lynn J, Dy SM, et al.: Evidence for improving palliative care at the end of life: A systematic review. Ann Intern Med 2008;148(2):147-159.

41. Higginson IJ, Finlay I, Goodwin DM, et al.: Do hospitalbased palliative teams improve care for patients or families at the end of life? J Pain Symptom Manage 2002;23(2): 96-106.

42. VanderWalde A: Clinical ethics case report: Questionable capacity and the guidance of living wills. J Clin Ethics 2011; 22(3):250-255.

43. Levi BH, Heverley SR, Green MJ: Accuracy of a decision aid for advance care planning: Simulated end-of-life decision making. J Clin Ethics 2011;22(3):223-238.

44. Gysels M, Higginson IJ: Improving Supportive and Palliative Care for Adults With Cancer: Research Evidence Manual. London: National Institute for Clinical Excellence, 2004. www.nice.org.uk/guidelines/. (Last accessed July 15, 2012.)

45. Ringdal GI, Jordhoy MS, Ringdal K, Kaasa S: The first year of grief and bereavement in close family members to individuals who have died of cancer. Palliat Med 2001;15(2): 91-105.

46. Pantilat SZ, O'Riordan DL, Dibble SL, Landefeld CS: Hospital-based palliative medicine consultation: A randomized controlled trial. Arch Intern Med 2010;170(22):2038-2040.

Address correspondence to: Sydney M. Dy, MD, MSc 624 N. Broadway Room 609 Baltimore, MD 21205

E-mail: sdy@jhsph.edu 
Appendix A: Detailed Search Strategy (All 2000-2011)

\begin{tabular}{ll}
\hline PubMed & (Cancer[tiab] AND care[tiab]) AND (communication[mh] OR communication[tiab] \\
& OR psychosocial[tiab] OR distress[tiab] OR (pain[tiab] AND management[tiab])) \\
NOT (editorial[pt] OR comment[pt]) & (Cancer AND Care) AND Communication OR psychosocial OR distress OR \\
PsychInfo & (Pain AND management) from 2000 to 2011 \\
1 & Cancer AND care \\
2 & Communication OR psychosocial OR distress \\
3 & Pain AND management \\
4 & S2 OR S3 \\
5 & S1 AND S4 \\
6 & S1 AND S4 from 2000 to 2011 \\
1 & Cancer AND care \\
2 & Communication MeSH \\
3 & (Cancer AND care) AND (Communication) \\
4 & Communication \\
5 & (Cancer AND care) AND (Communication MeSH OR Communication tiab) \\
6 & Psychosocial OR distress \\
7 & Pain MeSH \\
8 & Pain \\
9 & Management \\
10 & (Cancer AND care) AND (Communication MeSH OR Communication tiab OR psychosocial \\
11 & OR distress OR ((pain MeSH OR pain tiab) AND management)) \\
DARE & (Cancer AND care) AND (Communication MeSH OR Communication tiab OR psychosocial \\
& OR distress OR ((pain MeSH OR pain tiab) AND management)) from 2000-2011 \\
& Cancer AND care AND (communication OR psychosocial OR distress OR (pain AND management)) \\
\hline
\end{tabular}




\section{This article has been cited by:}

1. Sydney M. Dy, Nebras Abu Al Hamayel, Susan M. Hannum, Ritu Sharma, Sarina R. Isenberg, Kamini Kuchinad, Junya Zhu, Katherine Smith, Karl A. Lorenz, Arif H. Kamal, Anne M. Walling, Sallie J. Weaver. 2017. A Survey to Evaluate Facilitators and Barriers to Quality Measurement and Improvement: Adapting Tools for Implementation Research in Palliative Care Programs. Journal of Pain and Symptom Management 54:6, 806-814. [Crossref]

2. C. Udo, M. Lövgren, G. Lundquist, B. Axelsson. 2017. Palliative care physicians' experiences of end-of-life communication: A focus group study. European Journal of Cancer Care 51, e12728. [Crossref]

3. Kimberly K. Garner, Patricia Dubbert, Shelly Lensing, Dennis H. Sullivan, Rebecca A. Aslakson, Katherine Ast, Ronit Elk, Kimberly K. Garner, Robert Gramling, Corita Grudzen, Arif H. Kamal, Sangeeta Lamba, Thomas W. LeBlanc, Ramona L. Rhodes, Eric Roeland, Dena Schulman-Green, Kathleen T. Unroe. 2017. Concordance Between Veterans' Self-Report and Documentation of Surrogate Decision Makers: Implications for Quality Measurement. Journal of Pain and Symptom Management 53:1, 1-4. [Crossref]

4. Laurent Billot, Kate Corcoran, Alina McDonald, Gawaine Powell-Davies, Anne-Marie Feyer. 2016. Impact Evaluation of a SystemWide Chronic Disease Management Program on Health Service Utilisation: A Propensity-Matched Cohort Study. PLOS Medicine 13:6, e1002035. [Crossref]

5. Anne Fishman-Bosc, Enzo Leveau, Sylvie Crelerot-Klopfenstein, Stéphanie Gentile, Sébastien Colson. 2016. Clarification de concept : la coordination appliquée au domaine des soins. Revue Francophone Internationale de Recherche Infirmière 2:2, 77-86. [Crossref]

6. S. A. Josephson. 2016. Focusing on transitions of care: A change is here. Neurology: Clinical Practice 6:2, 183-189. [Crossref]

7. Laura A. Lathrop, Andrew W. Gottfried. 2015. Quality Palliative Care and the First Domain. Journal of Hospice \& Palliative Nursing 17:6, 517-523. [Crossref]

8. Myers Jeff, Krueger Paul, Webster Fiona, Downar James, Herx Leonie, Jeney Christa, Oneschuk Doreen, Schroder Cori, Sirianni Giovanna, Seccareccia Dori, Tucker Tara, Taniguchi Alan. 2015. Development and Validation of a Set of Palliative Medicine Entrustable Professional Activities: Findings from a Mixed Methods Study. Journal of Palliative Medicine 18:8, 682-690. [Abstract] [Full Text HTML] [Full Text PDF] [Full Text PDF with Links] [Supplemental Material]

9. Daniel Nyhan, Peter J. Pronovost. 2014. Rebecca A. Aslakson, M.D., Ph.D., Recipient of the 2014 Presidential Scholar Award. Anesthesiology 121:4, 692-694. [Crossref] 